\title{
Inclusive searches for squarks and gluinos in final states with no leptons with the ATLAS detector
}

Otilia Anamaria Ducu*, on behalf of the ATLAS Collaboration

Université de Montreal (CA)

Horia Hulubei National Institute of Physics and Nuclear Engineering, IFIN-HH, Bucharest (RO)

E-mail: oducuecern.ch

Despite the absence of experimental evidence, weak scale supersymmetry remains one of the best motivated and studied Standard Model extensions. This document summarises recent ATLAS results on inclusive searches for supersymmetric squarks and gluinos, including third generation squarks produced in the decay of gluinos. The searches involve final states containing jets, missing transverse momentum and no light leptons, taus or photons, and were performed with $p p$ collisions at a centre-of-mass energy of $13 \mathrm{TeV}$.

The European Physical Society Conference on High Energy Physics

5-12 July

Venice, Italy

${ }^{*}$ Speaker. 


\section{Introduction}

An excellent description of currently known phenomena of the Universe at the infinitesimal scale is given by the Standard Model (SM). The success of its predictive power has culminated in July 2012, when the last SM elementary particle, the Higgs boson, was discovered [1, 2]. Besides all its accomplishments, several unexplained experimental observations or theoretical problems exist with the SM and some hints of new physics are expected at the $\mathrm{TeV}$ scale.

Supersymmetry (SUSY) is one of the best-motivated extensions of the SM, and in its minimal realization (MSSM) it predicts new bosonic (fermionic) partners to SM fermions (bosons), as well as an additional Higgs doublet. If $R$-parity is conserved (RPC) the lightest supersymmetric particle (LSP) is stable and represents a possible dark-matter candidate. If instead $R$-parity is violated (RPV), the LSP could decay within the detector volume, softening the missing transverse momentum $\left(\mathbb{E}_{T}\right)$ spectrum. The scalar partners of right-handed and left-handed quarks, $\tilde{q}_{R}$ and $\tilde{q}_{L}$, can mix to form two mass eigenstates, $\tilde{q}_{1}$ and $\tilde{q}_{2}$, where $\tilde{q}_{1}$ denotes the lighter particle. The $\mathrm{RL}$ mixing effect is proportional to the corresponding SM fermion masses and therefore becomes important for the third generation. Large mixing can yield a bottom squark, $\tilde{b}_{1}$, and a top squark, $\tilde{t}_{1}$, mass eigenstates which are significantly lighter than other squarks. Superpartners of the charged and neutral electroweak and Higgs bosons also mix to produce charginos $\left(\tilde{\chi}_{1}^{ \pm}\right)$and neutralinos $\left(\tilde{\chi}^{0}\right.$, usually the LSPs). Squarks and the fermionic partners of the gluons, the gluinos ( $\tilde{g})$, could be produced in strong-interaction processes at the Large Hadron Collider (LHC) and decay via cascades, as illustrated in Figure 1.
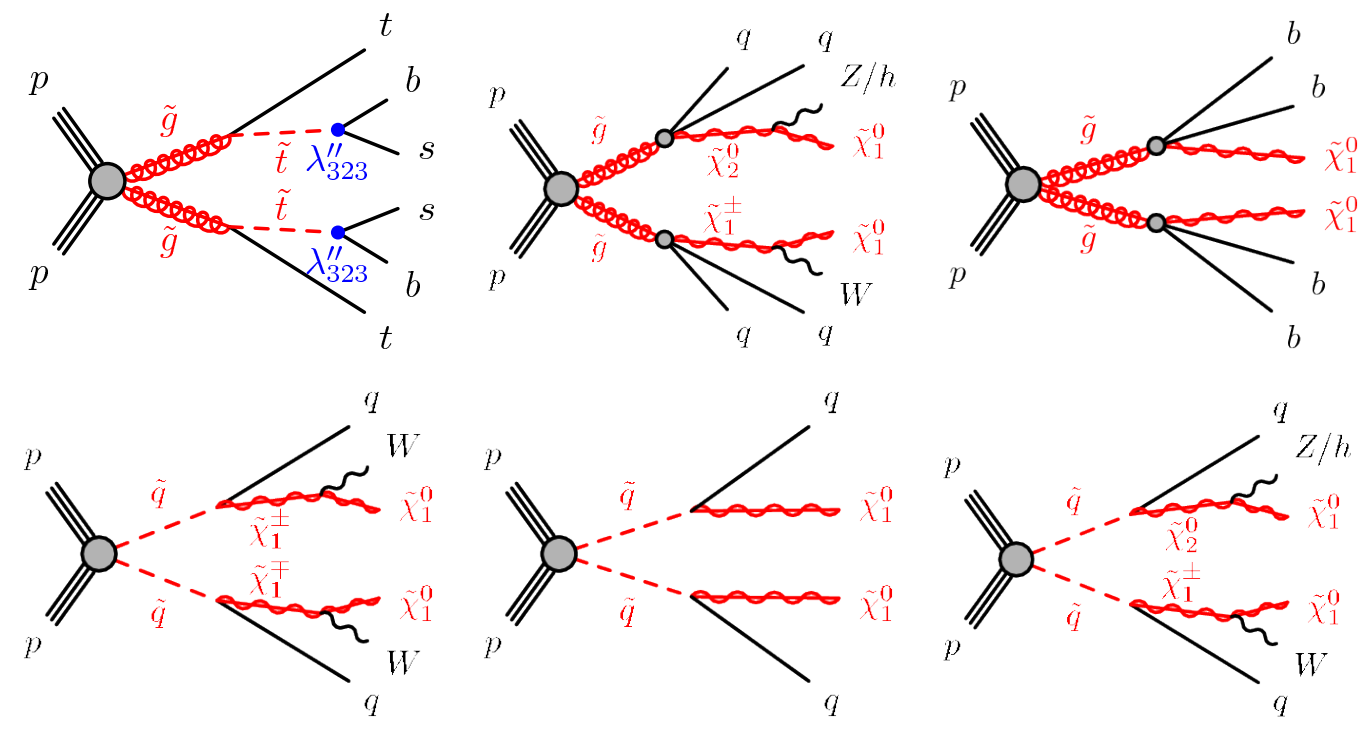

Figure 1: Example of decay topologies of squark-pair production and gluino-pair production in the SUSY simplified models.

This document presents a search for gluinos and squarks that utilizes final states with multiple jets and significant $\mathbb{E}_{T}$ observed by the ATLAS detector [3] at the LHC $[4,5,6]$. This search is conducted using $\sim 36 \mathrm{fb}^{-1}$ of proton-proton collisions at $\sqrt{s}=13 \mathrm{TeV}$ recorded in 2015 and 2016. Results are interpreted using SUSY simplified models, where the particles not involved in production or decays are decoupled. 


\section{Analysis strategy and background estimation}

To search for a possible signal, selections are defined to enhance the expected signal yield relative to the SM backgrounds. Signal regions (SRs) with no leptons in the event are defined using the Monte Carlo (MC) simulation of SUSY signals and the SM background processes, and are optimized to maximize the expected sensitivity to each model considered. A variety of signal regions are defined depending on e.g. jet multiplicity (in Ref. [4] some SRs require at least 10 jets in the event), mass of the large-radius jets [5, 6], effective mass $\left(\mathrm{m}_{\mathrm{eff}}\right), \mathbb{Z}_{T}$, etc.

In this search, the main SM backgrounds are $W / Z+$ jets, $t \bar{t}$, single top, di-boson and multi-jets processes. To estimate these backgrounds in an accurate and robust way, control regions (CRs) are defined for each of the signal regions and used to normalize the background MC simulation. The CRs are chosen to be orthogonal to the SR selections in order to provide independent data samples enriched in particular background sources. The selections are optimized to have negligible signal contamination, while minimizing the systematic uncertainties arising from the extrapolation of the $\mathrm{CR}$ event yields to estimate backgrounds in the SR. Figure 2 left illustrates the $\mathrm{m}_{\text {eff }}$ distribution in a typical CR targeting $W+$ jets processes, as defined in Ref. [6]. The good agreement between the observed data and the estimated SM background gives confidence in the employed technique. The multi-jets background is estimated using a data-driven technique. Cross-checks of the background estimates are performed with data in several validation regions (VRs) selected with requirements such that these regions are in-between the CR and SR selections, not overlapping, and also have a low expected signal contamination.
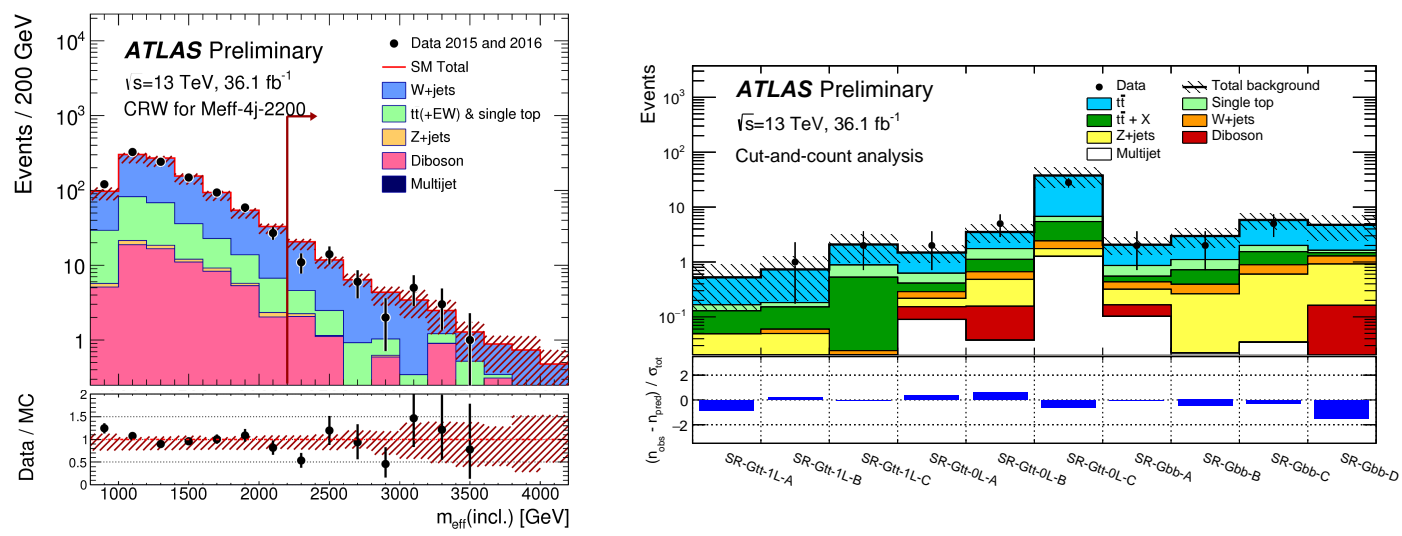

Figure 2: Left: $m_{\text {eff }}$ distributions in the Meff-CRW control region defined in Ref. [6] (enriched in $W+$ jets backgrounds). The arrows indicate the values at which the requirements on $\mathrm{m}_{\mathrm{eff}}$ are applied. The histograms denote the MC background expectations, normalized to cross-section times integrated luminosity and the dominant process in the $\mathrm{CR}$ is normalized to data. The hatched (red) error bands denote the combined experimental, MC statistical and theoretical modelling uncertainties. Right: Results in the SRs defined for the cut-and-count analysis documented in Ref. [5]. The upper panel shows the observed number of events and the predicted background yield. All experimental and theoretical uncertainties are included in the uncertainty band. The background category $t \bar{t}+X$ includes $t \bar{t}+W / Z, t \bar{t}+h$ and $t \bar{t} t \bar{t}$ events. The lower panel shows the pulls, defined by the difference between the observed number of events $\left(\mathrm{n}_{\mathrm{obs}}\right)$ and the predicted background yield $\left(\mathrm{n}_{\text {pred }}\right)$ divided by the total uncertainty $\left(\sigma_{\mathrm{tot}}\right)$, in each SR defined in Ref. [5]. 


\section{Results and conclusions}

Figure 2 right illustrates the results in the SRs defined for the cut-and-count analysis documented in Ref. [5]. No significant excess over the Standard Model prediction is observed and this is generally true for all the signal regions defined in Ref. $[4,5,6]$. Therefore, model-independent limits are set on the visible cross-section for new physics processes. Exclusion limits are also set on gluino (squark) and LSP masses using SUSY simplified models, as shown in Figure 3.
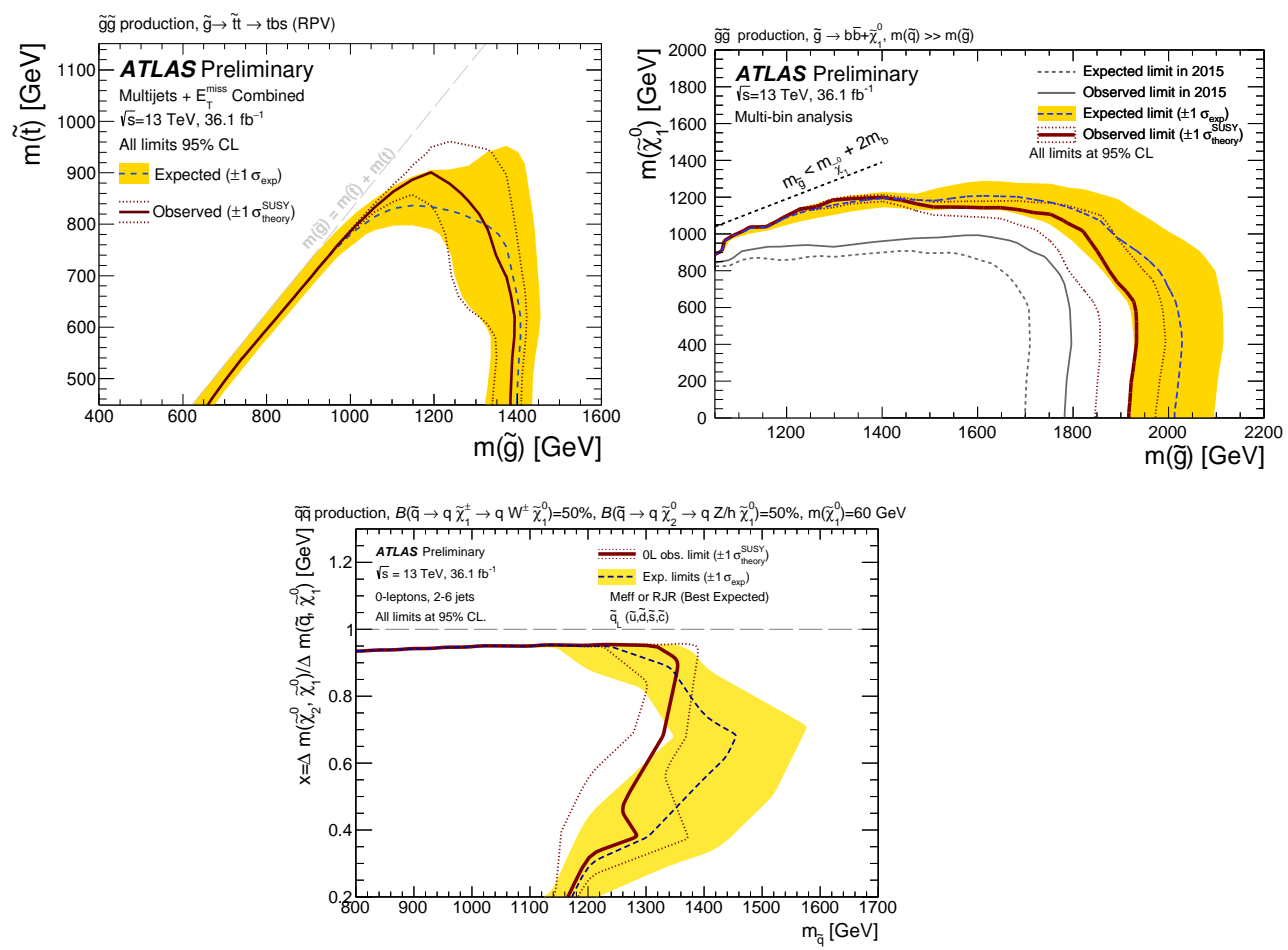

Figure 3: Exclusion contours in gluino or direct squark production in $R$-parity-violating (left) [4] and $R$ parity-conserving scenarios (middle and right) [5,6]. The solid maroon line indicates the observed limit, while the dashed blue line shows the expected limit. Experimental, MC theoretical and statistical uncertainties are shown in the yellow band. Dotted maroon lines delimit the variation of the observed limit within the $\pm 1 \sigma$ uncertainties on the signal cross-section.

Exclusion limits are set in an R-parity-violating model with baryon-number-violating couplings permitting $\tilde{t}_{1} \rightarrow \bar{s} \bar{b}$, such that the gluino mass must be greater than $1.25-1.35 \mathrm{TeV}$ when the top squark mass is in the range $0.4<m_{\tilde{t}_{1}}<0.8 \mathrm{TeV}$ [4]. When limits are set on gluino and LSP masses in $R$-parity-conserving simplified models where the gluino decays exclusively as $\tilde{g} \rightarrow b \bar{b} \tilde{\chi}_{1}^{0}$ or $\tilde{g} \rightarrow t \bar{\tau} \tilde{\chi}_{1}^{0}$, gluino masses of less than $1.92 \mathrm{TeV}$ and $1.97 \mathrm{TeV}$ are excluded at the 95\% CL for LSP masses below $\sim 0.3 \mathrm{TeV}$ [5]. For a simplified model involving the strong production of squarks of the first and second generations, with decays to a massless lightest neutralino, squark masses below $1.58 \mathrm{TeV}$ are excluded, assuming mass-degenerate squarks of the first two generations. In simplified models with pair-produced squarks and gluinos, each decaying via an intermediate $\tilde{\chi}_{1}^{ \pm}$to one quark or two quarks, a $W$ boson and a $\tilde{\chi}_{1}^{0}$, squark masses below $1.15 \mathrm{TeV}$ and gluino masses below $2.01 \mathrm{TeV}$ are excluded for massless $\tilde{\chi}_{1}^{0}[6]$.

These results substantially extend the region of supersymmetric parameter space excluded by previous LHC searches. 


\section{References}

[1] ATLAS Collaboration, Observation of a new particle in the search for the Standard Model Higgs boson with the ATLAS detector at the LHC, Phys.Lett. B 716 (2012) 1 [hep-th/12077214].

[2] CMS Collaboration, Observation of a new boson at a mass of $125 \mathrm{GeV}$ with the CMS experiment at the LHC, Phys.Lett. B 716 (2012) 30 [hep-th/12077235].

[3] ATLAS Collaboration, The ATLAS experiment at the CERN Large Hadron Collider, JINST 3 (2008) S08003

[4] ATLAS Collaboration, Search for new phenomena with large jet multiplicities and missing transverse momentum using large-radius jets and flavour-tagging at ATLAS in $13 \mathrm{TeV}$ pp collisions, ATLAS-CONF-2017-033, https: / / cds . cern. ch/record/2265802, (2017)

[5] ATLAS Collaboration, Search for production of supersymmetric particles in final states with missing transverse momentum and multiple b-jets at $\sqrt{s}=13 \mathrm{TeV}$ proton-proton collisions with the ATLAS detector, ATLAS-CONF-2017-021, https: / / cds.cern.ch/record/2258143/, (2017)

[6] ATLAS Collaboration, Search for squarks and gluinos in final states with jets and missing transverse momentum using $36 \mathrm{fb}^{-1}$ of $\sqrt{\mathrm{s}}=13 \mathrm{TeV}$ pp collision data with the ATLAS detector, ATLAS-CONF-2017-022, https: / / cds. cern. ch/record/2252643, (2017) 